\title{
TNFRSF21 participates in Streptococcus agalactiae- induced inflammatory necrosis through the NLRP3 inflammasome pathway
}

\section{Lu Yuan}

Anhui Normal University

Jian Ruan

Anhui Normal University

Mangling Zhang

Anhui Normal University

Xiaojun Jiang

Anhui Normal University

Jun Li

Anhui Normal University

Yafi Cai ( $\nabla$ ycai@njau.edu.cn )

Nanjing Agricultural University https://orcid.org/0000-0001-9057-7636

\section{Research Article}

Keywords: Streptococcus agalactiae, TNFRSF21, Pyroptosis, NLRP3 inflammasome

Posted Date: February 23rd, 2021

DOl: https://doi.org/10.21203/rs.3.rs-209964/v1

License: (1) This work is licensed under a Creative Commons Attribution 4.0 International License.

Read Full License 


\section{Abstract}

Bovine mastitis caused by Streptococcus agalactiae (S. agalactiae) has resulted in substantial economic loss to the animal husbandry industry; however, the associated molecular mechanism remains unclear and the role of TNFRSF21 in the inflammatory response caused by S. agalactiae is unknown. In this study, we focused on bacterial isolation and identification, histological observations, and provide the first report of the association between TNFRSF21 and pyroptosis. The results showed that $S$. agalactiae induced inflammation can cause increasing damage of the tissue and pyroptosis ratio, significantly increased the level of inflammatory cytokine production (e.g., NLRP3, caspase-1, and IL-1 $\beta$ ), and upregulate TNFRSF21 following $S$. agalactiae infection in a time-dependent manner. In conclusion, these findings indicated that it may fight against the inflammatory response induced by $S$. agalactiae by inhibiting TNFRSF21 expression and reducing NLRP3 inflammasome pathway-mediated inflammatory necrosis.

\section{Introduction}

Bovine mastitis is caused by inflammation of the udder tissue due to a microbial infection or physical trauma, which is responsible for substantial economic loss to the dairy industry [1,2]. Various pathogenic factors can induce mastitis, including the pasture environment, milking methods, bacteria, and host genotype [3-5]; however, an infection with pathogenic bacteria represents the main cause of mastitis. When pathogenic bacteria invade the mammary gland, the increased permeability of the blood-milk barrier gives rise to a high number of somatic cells in the milk [6]. At the same time, mammary epithelial cells and immune cells work together through pattern recognition receptors that detect pathogen-related molecular patterns to induce a rapid and powerful immune response, which may cause inflammation while removing pathogenic microorganisms $[7,8]$. At present, $S$. agalactiae has attracted increased attention due to its high prevalence, and associated risk of inter-species transmission [9], which can lead to a variety of diseases (e.g., dairy cow mastitis, neonatal meningitis, sepsis, and toxic shock) [10,11]. However, the mechanism of $S$. agalactiae induced inflammation remains poorly understand. Thus, this study used $S$. agalactiae to construct inflammatory models to elucidate the associated mechanism.

Tumor necrosis factor (TNF) receptors play an important role in the cellular resistance to pathogens and tumor-related immune response, and are key factors of both the inflammatory and immune regulatory responses $[12,14]$. As a member of the TNF receptor superfamily, TNFRSF21 is highly expressed in a variety of tumor tissues (e.g., ovarian cancer, breast cancer, and adult sarcoma) with potential anticancer effects and can promote the apoptosis of both untransformed and tumor-derived cells [13-15]. In addition, TNFRSF21 has been found to regulate the proliferation and differentiation of immune cells $[16,17]$. TNFRSF21 has also been shown to have a certain promotional effect on endothelial cell necrosis induced by lupus susceptible mice and autoimmunity caused by tumor cells $[18,19]$. The activation of nuclear factor-KB (NF-KB) can induce TNFRSF21 expression, the overexpression of which will lead to activation of the NF-KB pathway [15]. The signal mediated by NF-KB is necessary to maintain TNFRSF21 expression in Jurkat leukemia cells [20]. When TNFRSF21 is blocked, the NF-kB signaling pathway is also 
inhibited [21]. In contrast, the up-regulation of TNFRSF21 expression can induce HeLa apoptosis [22]. This suggests that TNFRSF21 may mediate the inflammatory responses in tumor progression via NF-KB $[23,24]$. Coincidentally, once NF-KB is activated, it can regulate the expression of inflammatory genes and cytokine release (e.g., TNF- $\alpha$, IL-1 $\beta$, and pro-caspase-1), which assemble on the scattered Golgi apparatus to form NLRP3 inflammasomes, leading to cell pyroptosis and lysis [25-28]. Under normal conditions, pyroptosis represents an important component of the immune response to resist various diseases [29]; however, excessive pyroptosis will inevitably lead to the occurrence of systemic sepsis [30]. Our previous research found that TNSFRSF21 genetic polymorphisms are significantly related to the susceptibility and tolerance of dairy cow mastitis through a genome-wide association analysis [31]. However, the role of TNFRSF21 in the occurrence and development of mastitis remains unknown.

In this study, a murine mastitis model of $S$. agalactiae infection was constructed and we found that NLRP3 was significantly increased and accompanied by upregulated TNFRSF21 expression, indicating that NLRP3 may promote TNFRSF21 expression during the inflammatory response of the breast tissue and jointly promoted pyroptosis. Thus, TNFRSF21 may represent a novel drug target for regulating cellular pyroptosis and relieving inflammation.

\section{Materials And Methods}

\section{Milk sample collection}

A total of 20 milk samples, including eight normal milk samples and 12 mastitis milk samples, which were collected from the Weigang Animal Diary Company, Jiangsu, China.

\section{Bacterial strains}

S. agalactiae was isolated from fresh milk samples collected from cows with mastitis and injected into the mammary gland tissue of mice to construct a mastitis model. A single colony was isolated from the fresh milk samples using the plate-drawing method and expanded culture to extract the bacterial DNA. After sequencing, multiple pathogenic bacteria (e.g., S. agalactiae) were obtained (primer sequence: F5'AGTTTGATCCTGGCTCAG-3' and R5'-AGGCCCGGGGAACGTATTCAC-3', Sangon Biotech, Shanghai, China). S. agalactiae was incubated on a shaker for $12 \mathrm{~h}\left(37^{\circ} \mathrm{C}\right.$ at $\left.220 \mathrm{rpm}\right)$, diluted with PBS, and coated on the plate to achieve the desired concentration $\left(1 \times 10^{9} \mathrm{CFU} / \mathrm{mL}\right)$, in accordance with previously published methods [27]. The bacteria was stored for a short time at $4^{\circ} \mathrm{C}$. Before use, $1 \mathrm{~mL}$ of the diluted bacterial solution was resuscitated for $4 \mathrm{~h}\left(37^{\circ} \mathrm{C}\right.$ at $\left.220 \mathrm{rpm}\right)$, and centrifuged for $5 \mathrm{~min}(3000 \mathrm{rpm})$. The supernatant was removed and the bacterial pellet was resuspended in $1 \mathrm{~mL}$ PBS.

\section{Animals}

A total of 36 female and 15 male KM mice (6-8-weeks-old, weighing $20 \mathrm{~g}-25 \mathrm{~g}$ ) were maintained under SPF housing conditions. Both the female and male mice cohabited at a two female to one male ratio for conception, standard rearing, and free drinking water $\left(25^{\circ} \mathrm{C} \pm 1^{\circ} \mathrm{C}\right.$, humidity $\left.40 \%-80 \%\right)$. They were housed 
separately after pregnancy until 12 days after calving. The feeding and management of the experimental animals were carried out in accordance with the Animal Management Regulations issued by the National Science and Technology Commission of the People's Republic of China (November 14, 1988), and with the permission of the Animal Ethics Committee of Anhui Normal University (Approval No. 2015002).

\section{Mouse model of mastitis}

Female mice were randomly separated into two groups: 1) S. agalactiae group, one breast was injected with PBS and the other was injected with $S$. agalactiae after 12 days post-partum; and 2) PBS group, one breast received a blank control and the other was injected with PBS after 12 days post-partum. The pups were removed $2 \mathrm{~h}$ prior to the experiment. The female mice were anesthetized with $1 \%$ carbrital, and the third and fourth pairs of milk areas were wiped with a cotton ball soaked in $75 \%$ alcohol. Slowly, $100 \mu \mathrm{L}$ of $S$. agalactiae was injected into the left breast area with a microinjector needle with a diameter of 0.06 $\mathrm{mm}$, and $100 \mu \mathrm{L}$ of sterile PBS was injected into the right side. At the corresponding time points $(24 \mathrm{~h}, 48$ $\mathrm{h}$, and $72 \mathrm{~h}$ ) after treatment, the mice were sacrificed by cervical dislocation, and the mouse mammary glands were collected under aseptic conditions for subsequent processing and analysis.

\section{Histopathological analysis}

The mammary gland samples of the S. agalactiae group and PBS group were collected and fixed in $4 \%$ buffered paraformaldehyde for more than $48 \mathrm{~h}$. the samples were embedded in paraffin, sectioned, and stained with hematoxylin and eosin (H\&E), and the pathological changes were observed by microscopy.

\section{Western blot analysis}

After mixing $400 \mu \mathrm{L}$ cell lysate and $4 \mu \mathrm{L}$ proteinase inhibitor, $40 \mathrm{mg}$ of udder tissue were added, ground for $50 \mathrm{~s}$ into a powder on an ultrasonic shaker, and lysed for $2.5 \mathrm{~h}$ on a shaker. The lysate was centrifuged at $13,000 \times g$ for 20 min and the entire process was carried out at $4^{\circ} \mathrm{C}$. Next, the supernatant was collected into a new centrifuge tube and the supernatant was quantified using a BCA Protein Assay Kit (Beyotime Biotechnology, Shanghai, China). An appropriate amount of SDS-PAGE loading buffer was added to the protein sample and placed into a metal incubator for $95^{\circ} \mathrm{C} 10 \mathrm{~min}$. The samples were allowed to cool to room temperature and stored at $-20^{\circ} \mathrm{C}$. After the protein was transferred to the PVDF membrane, it was blocked in $5 \% \mathrm{BSA}$ for $1 \mathrm{~h}$ and incubated with the primary antibody at $4^{\circ} \mathrm{C}$ overnight. The membranes were then washed three times with TBST for $10 \mathrm{~min} /$ wash. The membranes were incubated with a horseradish peroxidase-labeled secondary antibody at $37^{\circ} \mathrm{C}$ for $1 \mathrm{~h}$. After washing the membrane three times with TBST, an enhanced chemiluminescence solution detection system was used to take pictures and perform an analysis (Tano, Shanghai, China). Densitometry was performed using ImageJ software (National Institutes of Health, Bethesda, MD). The following antibodies were used in this study: TNFRSF21 (Catalog No: \#bs-7678R, BOSTER Biotechnology, Wuhan) 68 kDa, NLRP3 (Catalog No: \#sc-134306, Santa Cruz Biotechnology, USA) 120 kDa, IL-1 (Catalog No: \#sc-12742, Santa Cruz Biotechnology, USA) 17 kDa, caspase1 (Catalog No: \#sc-392736, Santa Cruz Biotechnology, USA) 45 kDa, GAPDH (Catalog No: \#AT0002, CMCTAG, USA) 36 kDa, goat anti-mouse IgG secondary antibody (Catalog 
No: \#BA1038, BOSTER Biotechnology, Wuhan), and goat anti-rabbit IgG secondary antibody (Catalog No: \#BA1039, B0STER Biotechnology, Wuhan).

\section{Pyroptosis detection}

Mammary tissue sections (thickness: $4 \mu \mathrm{m}$ ) were deparaffinized and dehydrated by density and fluorescently stained. Densitometry was performed using ImageJ software (National Institutes of Health, Bethesda, MD). The methods were performed in accordance with the manufacturer instructions in the TUNEL cell pyroptosis detection kit-CY3 (50T) (BOSTER, Wuhan, China).

\section{Statistical analysis}

Statistical analyses were performed using a Student's $t$-test with GraphPad Prism version 6.0 (GraphPad Software, San Diego, CA, USA). Data were presented as the mean \pm the standard deviation (SD). The fluorescence intensity was determined by defining a circular region-of-interest (ROI) for the entire cell. ROIs were corrected based on the average value of a background ROI defined outside the cell. $\mathrm{A}$ colocalization analysis was performed using JACoP Plugin in ImageJ. A threshold of $P<0.05$ was considered statistically significant.

\section{Results}

\section{Isolation and identification of $S$. agalactiae}

S. agalactiae is an epidemic zoonotic pathogen that was initially detected in dairy products. We isolated six strains from 50 single colonies in fresh milk samples by $16 \mathrm{~S}$ rRNA sequencing, including $S$. agalactiae, Escherichia coli, Staphylococcus aureus, Bacillus subtilis, Pseudomonas aeruginosa, and Klebsiella pneumoniae. For further confirmation, we cultured Streptococcus in a blood Agar medium exhibiting gray colonies (Fig. 1a). The bacteria were Gram-stained, revealing a purple chain shape (Fig.1b). PCR detection was developed based on -1,4-glusidase gene and the amplified fragment was approximately $1283 \mathrm{bp}$ (Fig.1c). The recovered and purified PCR products were sent for sequencing (Sangon Biotech, Shanghai, China). The maximum similarity of the 16S rRNA sequence between this strain (CSA 001) and S. agalactiae (NR 117503.1) was 99.85\%, they were located on the same evolutionary branch, and the Bootstrap value was $100 \%$ (Fig.1d).

\section{S. agalactiaeinduced histopathological impairment of the mammary gland in mice.}

Mice were sacrificed at $24 \mathrm{~h}, 48 \mathrm{~h}$, and $72 \mathrm{~h}$ after the mammary glands were injected with $\mathbf{S}$. agalactiae. After dissection, the third and fourth pairs of the breast areas were full of milk at $24 \mathrm{~h}$, and no obvious inflammation was observed. At $48 \mathrm{~h}$, the mammary gland areas became swollen and purple. After $72 \mathrm{~h}$, the mammary gland areas on both sides were severely congested, the breast was degenerated, and pus had obviously appeared on the left side (S. agalactiae injection) (Fig. 2). We further fixed the breast tissues, cut them into sections, and stained with H\&E. While the acinus in the control group was found to be relatively intact, the acini in the $\boldsymbol{S}$. agalactiae injection group displayed progressive damage with the 
extension of infection time. Furthermore, the injury was more obvious in the breast tissue in the $\boldsymbol{S}$. agalactiae treatment group compared with the control group. The epithelial cells had sloughed off the acinar structure was gradually destroyed, interstitial thickening was observed, and immune cell infiltration, neutrophils, and exfoliated necrotic breast epithelial cells were scattered in the acinar lumen. Over time, these phenomena became increasingly more serious (Fig.2).

\section{S. agalactiae increased the level of IL-1 $\beta$, NLRP3, caspase-1, and TNFRSF21 expression in the mouse mammary glands.}

To explore the role of TNFRSF21 in a mouse mastitis model induced by $S$. agalactiae, we detected the level of TNFRSF21, NLRP3, IL-1 $\beta$, and caspase-1 protein expression by Western blot. From left to right, Figures $3 a$ and e show the expression for the normal 1, normal 2, control, and treatment groups. Normal 1 and normal 2 were from the same pregnant mouse after stopping breastfeeding for $2 \mathrm{~h}$, with one breast as the blank control and the other injected with PBS in the third pair, which was sacrificed at $24 \mathrm{~h}, 48 \mathrm{~h}$, and $72 \mathrm{~h}$ following PBS injection. The Control and treatment groups were from the same pregnant mouse that had stopping breastfeeding for $2 \mathrm{~h}$, with one breast injected with PBS and the other one injected with $S$. agalactiae in the third pair, which was sacrificed at $24 \mathrm{~h}, 48 \mathrm{~h}$, and $72 \mathrm{~h}$ after injection, respectively. We found that the level of TNFRSF21, NLRP3, IL-1 $\beta$, and caspase-1 expression in the normal 1 and normal 2 mice did not change significantly. Compared with the normal group, the level of TNFRSF21 expression in the control group did not change at $24 \mathrm{~h}$, but was higher at $48 \mathrm{~h}$ and $72 \mathrm{~h}(\mathrm{P}<0.01)$, and showed a significant upward trend from $24 \mathrm{~h}$ to $72 \mathrm{~h}$. The level of TNFRSF21 expression was significantly higher in the $S$. agalactiae treatment group than in the control group at $48 \mathrm{~h}$ and $72 \mathrm{~h}(\mathrm{P}<0.01)$ (Fig.3b). The level of NLRP3 expression in the treatment group peaked and was extremely significantly different with the other three groups at $24 \mathrm{~h}(\mathrm{P}<0.001)$. There was a subsequent downward trend in the level of NLRP3 expression, with an increasingly smaller difference, which was higher compared with the other three groups at any given time $(P<0.01)$. The level of NLRP3 in the control group peaked at $48 \mathrm{~h}$ and were higher than the normal group at $24 \mathrm{~h}, 48 \mathrm{~h}$, and $72 \mathrm{~h}(\mathrm{P}<0.05)$ (Fig.3c). The level of IL-1 $\beta$ expression in the control group increased substantially and was higher than that of the normal 1 and normal 2 groups at $24 \mathrm{~h}, 48 \mathrm{~h}$, and $72 \mathrm{~h}(\mathrm{P}<0.05)$; however, the treatment group expressed the highest level of IL-1 $\beta$ at 48 $h$, and the difference between the control group gradually became smaller, but remained at significant levels $(P<0.05)$ (Fig.3d). Compared with the normal 1 and normal 2 groups, the level of caspase- 1 expression in the control and treatment groups did not change significantly at $24 \mathrm{~h}$ and peaked at $48 \mathrm{~h}(\mathrm{P}$ $<0.01$ ). Although the level of caspase-1 expression in the control and treatment groups decreased at $72 \mathrm{~h}$, it remained at a higher level than that of the normal group $(P<0.05)$ (Fig.3f).

\section{S. agalactiae triggers pyroptosis in mouse mammary epithelial cells.}

To further explain whether the inflammatory response of the breast epithelial cells caused by $S$. agalactiae promotes TNFRSF21-mediated cell pyroptosis, we examined pyroptosis by TUNEL staining. (Fig. 4a, the arrows indicate pyroptosis). These results were confirmed by western blot. With an increase in infection time, the negative control group exhibited potential mammary epithelial cell pyroptosis. The 
mammary gland cellular pyroptosis ratios in the $\boldsymbol{S}$. agalactiae treatment group gradually increased, and were significantly higher than that of the control group $(P<0.001$; Fig. $4 b)$.

\section{Discussion}

Due to the diversity and complexity of its pathogenic factors, mastitis has been plaguing the development of global animal husbandry for several years, which is threating to human health. Recently, it has been discovered that $S$. agalactiae can frequently cause mastitis due to its highly infectious and pathogenic characteristics, [10], clinical neonatal infections, meningitis, endocarditis, and bacteremia [32]. Regarding the pathogenic molecular mechanism of mastitis caused by $S$. agalactiae, the experts' views are highly variable, and no conclusions have been reached. Our previous research found that TNSFRSF21 genetic polymorphisms are significantly related to the susceptibility and tolerance of dairy cow mastitis through a genome-wide association analysis [31]; however, the role of TNFRSF21 in S. agalactiae-induced mastitis remains unknown. In this study, we found that the mammary gland tissues of the mice infected with $S$. agalactiae (bacterial solution with a concentration of $1 \times 10^{9} \mathrm{CFU} / \mathrm{mL}$ ) displayed more severe histopathological damage than that of the control groups, and the level of caspase-1, IL-1 $\beta$, NLRP3, and TNFRSF21 expression was significantly increased. Interestingly, the control groups also displayed a considerable degree of inflammation.

NLRP3 inflammasomes can be detected in many inflammatory diseases, which leads to caspase-1 and IL-1 $\beta$ production [33]. Our results are consistent with studies that have shown that an $S$. aureus infection can trigger NLRP3 production [34]. As a Gram-positive bacterium, $S$. agalactiae can also induce high levels of NLRP3 expression, which is accompanied by a surge of IL-1 $\beta$. These cellular inflammatory factors can cause damage to the breast tissue [35]. Therefore, hematoxylin-eosin staining (H\&E) revealed that the mammary tissues of mice infected with $S$. agalactiae were more severely damaged than that of the control groups. However, the control groups also showed relatively severe damage and up-regulation of inflammatory factors, which may be caused by systemic infection. When there is an imbalance between the normal pyroptosis ratio and recovery ratio, it may lead to decreased barrier integrity, inducing bacterial translocation and the development of systemic sepsis [30].

TNFRSF21 up-regulation, T cell failure, and pyroptosis signals can be observed in patients with viral hepatitis [36]. Although the expression of pyroptosis signals is crucial for the removal of pathogens, an excessive stress response will cause continuous damage [37]. Our results showed that NLRP3, caspase-1, and IL-1 $\beta$ expression was significantly up-regulated during the early stages of infection. However, H\&E staining revealed that there was no obvious damage in mouse mammary tissues and PBS group infected by $S$. agalactiae at $24 \mathrm{~h}$. Furthermore, TNFRSF21 was not significantly up-regulated at $24 \mathrm{~h}$ following infection, which may represent the initial stage of the infection when the cell can still repair itself. After 48 h of infection, TNFRSF21 expression in both the control group and $S$. agalactiae treatment group showed a significant trend towards up-regulation, and the up-regulation of TNFRSF21 induced by S. agalactiae in the breast tissue was more obvious compared with the normal group. At the same time, the fluorescent pyroptosis staining results were also consistent with these findings. Both the control group and the $S$. 
agalactiae stimulation group had no obvious pyroptosis at $24 \mathrm{~h}$; however, the pyroptosis ratio was significantly increased after $48 \mathrm{~h}$. It has been revealed that the cells may enter a developmental phase of pyroptosis during the later stages of infection. It is during these later stages when the immune system is unable to repair the cells that have been over-damaged by the inflammatory response. TNFRSF21 expression was significantly up-regulated, which promoted cellular pyroptosis and tissue necrosis together with NLRP3.

\section{Conclusion}

In this study, S. agalactiae isolated from fresh milk samples was used to establish a mouse model of mastitis in this study. The findings of this study emphasized that $S$. agalactiae induced pyroptosis in mammary epithelial cells in vivo, and further promoted TNFRSF21 up-regulation through activation of the NLRP3 inflammasome and IL-1 $\beta$ expression, which aggravates tissue damage. However, there was no evidence that TNFRSF21 was a direct factor that led to pyroptosis in the later stages of the NLRP3 inflammasome. Further understanding of the role of $S$. agalactiae in the pathogenesis of mastitis will provide clues for the development of new treatment strategies to prevent disease.

\section{Declarations}

Funding This experiment was supported by The Agricultural Innovation fund of Jiangsu Province [grant no. CX (18)3014]; National Key R\&D Program of China (grant No. 2018YFC1200201); National Natural Science Foundation of China (grant No. 31970413); Anhui Natural Science Foundation (grant No. 1908085MC83); and Start-up grant from Nanjing Agricultural University (grant No. 804090).

Conflict of interest All authors declare that they have no conflict of interest.

Availability of data and material The datasets used or analysed during the current study are available from the corresponding author on reasonable request.

Ethics approval The experimental protocol was established, according to the ethical guidelines of the Declaration and was approved by the Ethics Committee of the Animal Management Regulations issued by the National Science and Technology Commission of the People's Republic of China (November 14, 1988), and with the permission of the Animal Ethics Committee of Anhui Normal University (Approval No. 2015002).

Consent to participate Not applicable.

Consent for publication Not applicable.

\section{References}


1. Heikkila, A.M., J.I. Nousiainen, and S. Pyorala, Costs of clinical mastitis with special reference to premature culling. J Dairy Sci, 2012. 95(1): p. 139-50.

2. Deb, R., et al., Trends in diagnosis and control of bovine mastitis: a review. Pak J Biol Sci, 2013. 16(23): p. 1653-61.

3. De Vliegher, S., et al., Invited review: Mastitis in dairy heifers: nature of the disease, potential impact, prevention, and control. J Dairy Sci, 2012. 95(3): p. 1025-40.

4. Klaas, I.C. and R.N. Zadoks, An update on environmental mastitis: Challenging perceptions. Transbound Emerg Dis, 2018. 65 Suppl 1: p. 166-185.

5. Gunther, J., et al., Comparison of the pathogen species-specific immune response in udder derived cell types and their models. Vet Res, 2016. 47: p. 22.

6. Wellnitz, O., et al., Short communication: Differential loss of bovine mammary epithelial barrier integrity in response to lipopolysaccharide and lipoteichoic acid. J Dairy Sci, 2016. 99(6): p. 48514856.

7. Takeda, K. and S. Akira, Toll-like receptors in innate immunity. Int Immunol, 2005. 17(1): p. 1-14.

8. Mifsud, E.J., A.C. Tan, and D.C. Jackson, TLR agonists as modulators of the innate immune response and their potential as agents against infectious Disease. Front Immunol, 2014. 5: p. 79.

9. Foxman, B., et al., Risk factors for group B streptococcal colonization: potential for different transmission systems by capsular type. Ann Epidemiol, 2007. 17(11): p. 854-62.

10. Pang, M., et al., Molecular and virulence characterization of highly prevalent Streptococcus agalactiae circulated in bovine dairy herds. Vet Res, 2017. 48(1): p. 65.

11. Sunkara, B., et al., Group B Streptococcus infections in non-pregnant adults: the role of immunosuppression. Int J Infect Dis, 2012. 16(3): p. e182-6.

12. Ward-Kavanagh, L.K., et al., The TNF receptor superfamily in co-stimulating and co-inhibitory responses. Immunity, 2016. 44(5): p. 1005-19.

13. Stegmann, S., et al., Death receptor 6 (DR6) is overexpressed in astrocytomas. Anticancer Res, 2019. 39(5): p. 2299-2306.

14. Pan, G., et al., Identification and functional characterization of DR6, a novel death domain-containing TNF receptor. FEBS Lett, 1998. 431(3): p. 351-6.

15. Kasof, G.M., et al., Tumor necrosis factor-alpha induces the expression of DR6, a member of the TNF receptor family, through activation of NF-kappaB. Oncogene, 2001. 20(55): p. 7965-75.

16. DeRosa, D.C., et al., Tumor-derived death receptor 6 modulates dendritic cell development. Cancer Immunol Immunother, 2008. 57(6): p. 777-87.

17. Schmidt, C.S., et al., Enhanced B cell expansion, survival, and humoral responses by targeting death receptor 6. J Exp Med, 2003. 197(1): p. 51-62.

18. Strilic, B., et al., Tumour-cell-induced endothelial cell necroptosis via death receptor 6 promotes metastasis. Nature, 2016. 536(7615): p. 215-8. 
19. Fujikura, D., et al., Death receptor 6 contributes to autoimmunity in lupus-prone mice. Nat Commun, 2017. 8: p. 13957.

20. Klima, M., et al., T-cell activation triggers death receptor-6 expression in a NF-kappaB and NF-AT dependent manner. Mol Immunol, 2011. 48(12-13): p. 1439-47.

21. Zhang, D., et al., MiR-210 inhibits NF-kappaB signaling pathway by targeting DR6 in osteoarthritis. Sci Rep, 2015. 5: p. 12775.

22. Dong, Y., et al., Lysophosphatidic acid triggers pyroptosis in HeLa cells through the upregulation of tumor necrosis factor receptor superfamily member 21. Mediators Inflamm, 2017. 2017: p. 2754756.

23. Yang, X., et al., Death receptor 6 (DR6) is required for mouse B16 tumor angiogenesis via the NFkappaB, P38 MAPK and STAT3 pathways. Oncogenesis, 2016. 5: p. e206.

24. Cui, L., Y. Han, and Z. Dong, Original paper miR-106a mimics the nuclear factor-KB signalling pathway by targeting DR6 in rats with osteoarthritis. Archives of Medical Science, 2020.

25. Wang, J.E., et al., Peptidoglycan and lipoteichoic acid from Staphylococcus aureus induce tumor necrosis factor alpha, interleukin 6 (IL-6), and IL-10 production in both T cells and monocytes in a human whole blood model. Infect Immun, 2000. 68(7): p. 3965-70.

26. Bi, C.L., et al., Selenium inhibits Staphylococcus aureus-induced inflammation by suppressing the activation of the NF-kappaB and MAPK signalling pathways in RAW264.7 macrophages. Eur J Pharmacol, 2016. 780: p. 159-65.

27. Breyne, K., et al., Efficacy and safety of a bovine-associated Staphylococcus aureus phage cocktail in a murine model of mastitis. Front Microbiol, 2017. 8: p. 2348.

28. Chen, J. and Z.J. Chen, Ptdlns4P on dispersed trans-Golgi network mediates NLRP3 inflammasome activation. Nature, 2018. 564(7734): p. 71-76.

29. Peterson, L.W., et al., RIPK1-dependent pyroptosis bypasses pathogen blockade of innate signaling to promote immune defense. J Exp Med, 2017. 214(11): p. 3171-3182.

30. Hunter, C.J., et al., Enterobacter sakazakii enhances epithelial cell injury by inducing pyroptosis in a rat model of necrotizing enterocolitis. J Infect Dis, 2008. 198(4): p. 586-93.

31. Yang, F., et al., Three novel players: PTK2B, SYK, and TNFRSF21 were identified to be involved in the regulation of bovine mastitis susceptibility via GWAS and post-transcriptional analysis. Front Immunol, 2019. 10: p. 1579.

32. Lyhs, U., et al., Streptococcus agalactiae Serotype IV in humans and cattle, Northern Europe(1). Emerg Infect Dis, 2016. 22(12): p. 2097-2103.

33. Mangan, M.S.J., et al., Targeting the NLRP3 inflammasome in inflammatory diseases. Nat Rev Drug Discov, 2018. 17(9): p. 688.

34. Zhao, G., et al., Polydatin reduces Staphylococcus aureus lipoteichoic acid-induced injury by attenuating reactive oxygen species generation and TLR2-NFkappaB signalling. J Cell Mol Med, 2017. 21(11): p. 2796-2808. 
35. Zhao, X. and P. Lacasse, Mammary tissue damage during bovine mastitis: causes and control. J Anim Sci, 2008. 86(13 Suppl): p. 57-65.

36. Barathan, M., et al., Chronic hepatitis $C$ virus infection triggers spontaneous differential expression of biosignatures associated with $T$ cell exhaustion and pyroptosis signaling in peripheral blood mononucleocytes. Pyroptosis, 2015. 20(4): p. 466-80.

37. Sadek, K., E. Saleh, and M. Ayoub, Selective, reliable blood and milk bio-markers for diagnosing clinical and subclinical bovine mastitis. Trop Anim Health Prod, 2017. 49(2): p. 431-437.

\section{Figures}




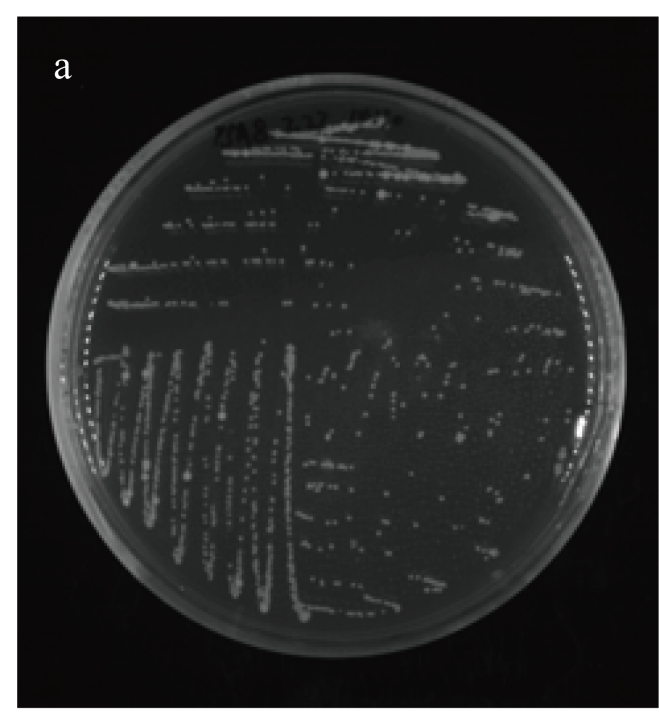

d

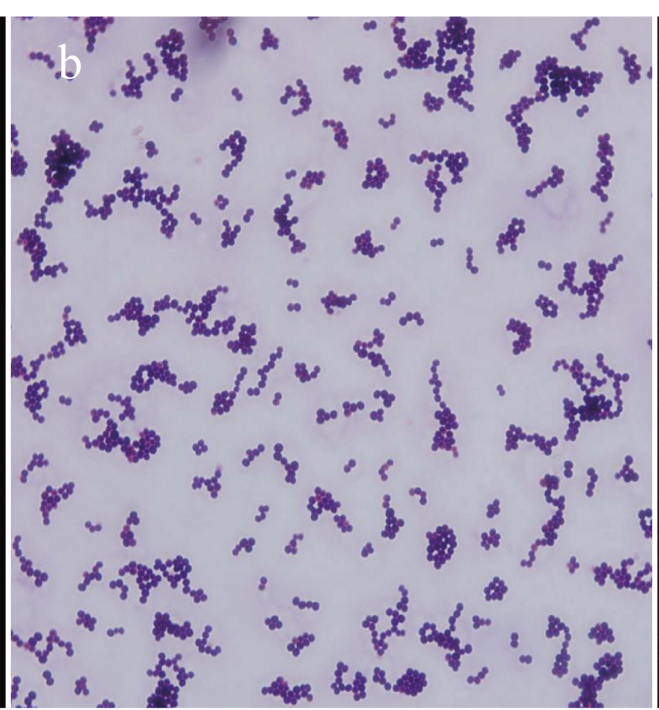

Streptococcus iniae (NR 115731.1)

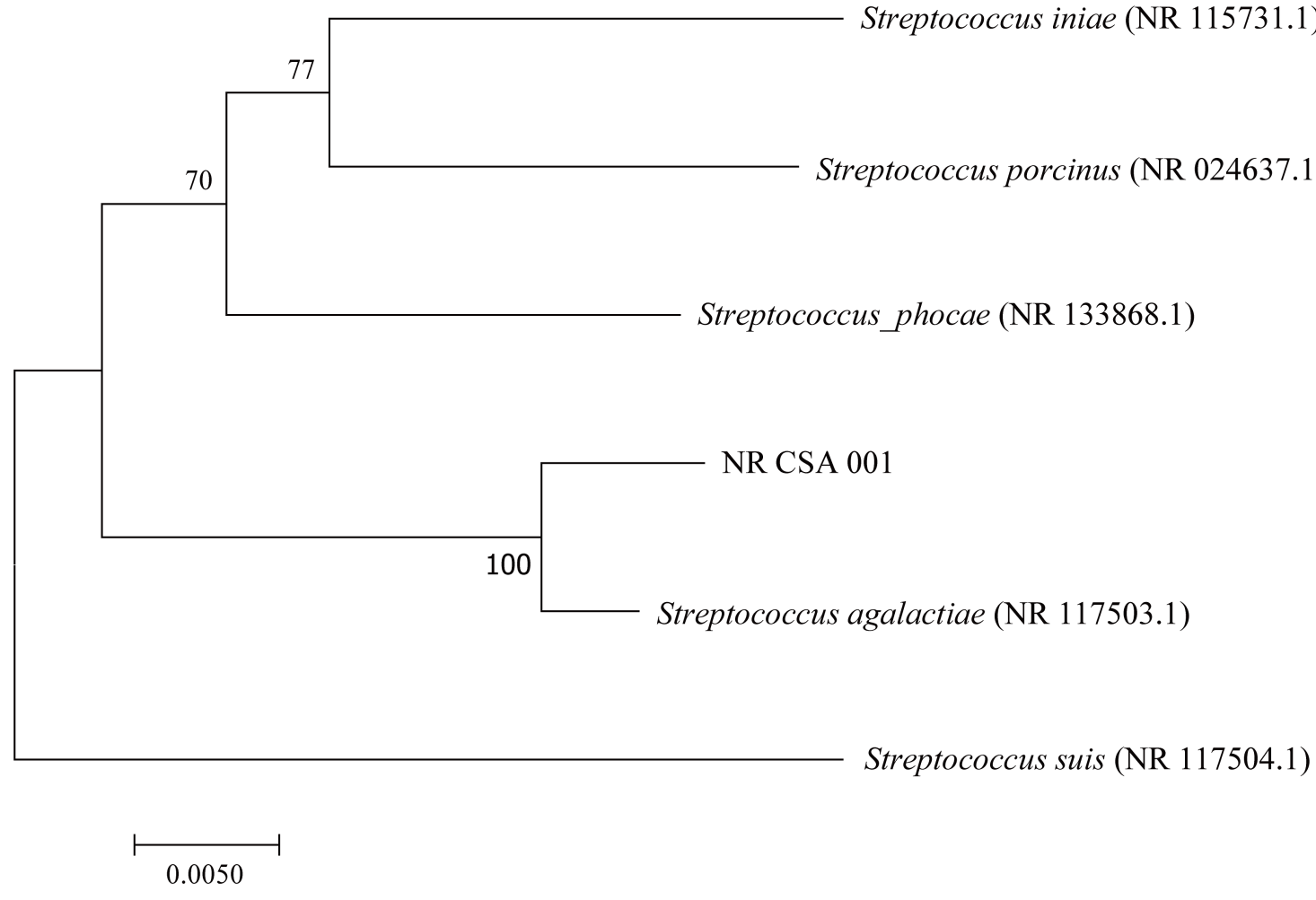

\section{Figure 1}

Isolation and identification of S. agalactiae. Identification of S. agalactiae: a The grayish-white colonies were grown in Colombian blood Agar culture medium. b Gram staining revealed purple chain bacteria. c A PCR assay was conducted based on the a-1,4-glucosidase gene, which was positive (1283 bp). d Sequencing: bacteria CSA 001 (1283 bp) and S. agalactiae (NR 117503.1) were the same bacteria, with a confidence level of $100 \%$. 


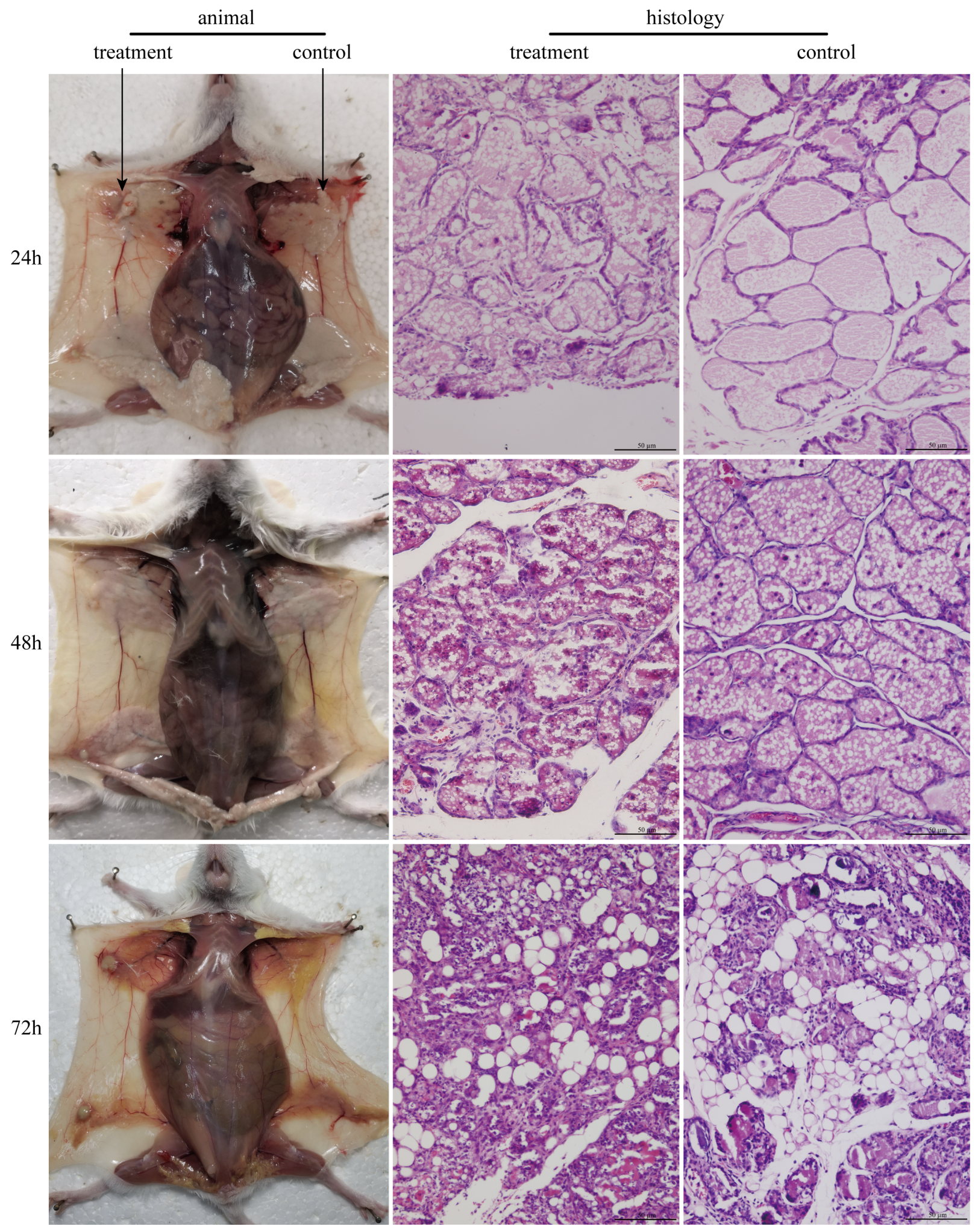

Figure 2

S. agalactiae-induced histopathological impairment in mice. S. agalactiae was injected into the third and fourth pair of mammary glands on the left, and PBS was injected into the third and fourth pair of mammary glands on the right. H\&E staining of breast histology: control group and treatment group (injected with S. agalactiae). 
a

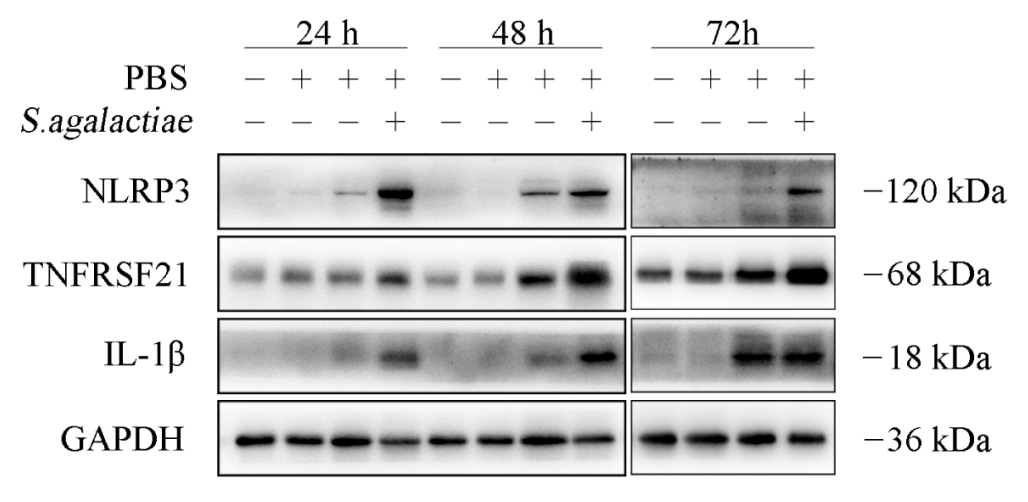

c

NLRP3

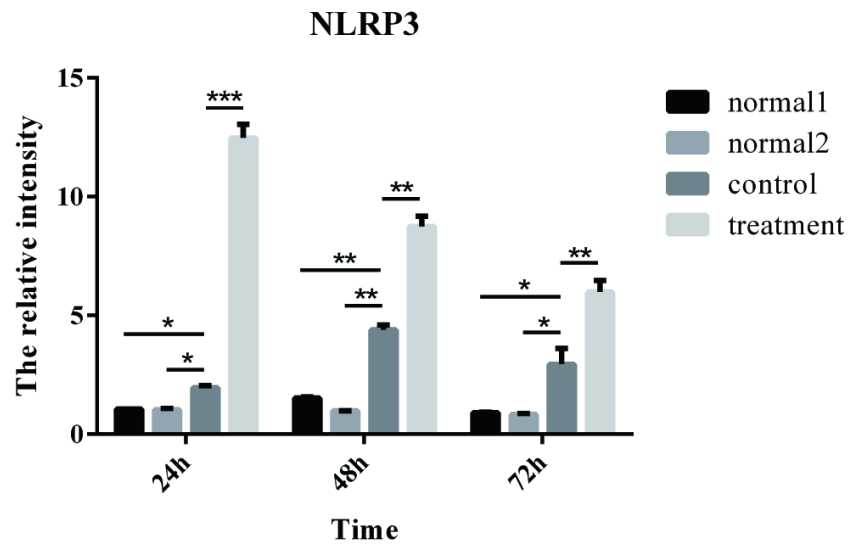

e

b

f

\section{TNFRSF21}

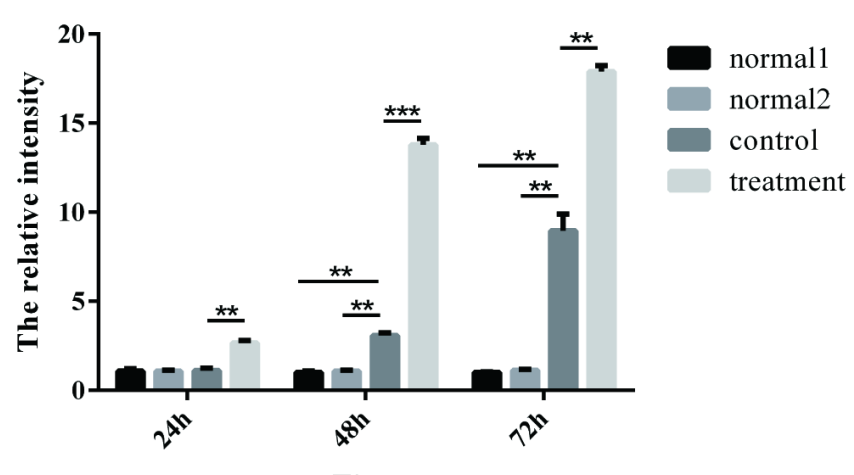

IL-1 $\beta$
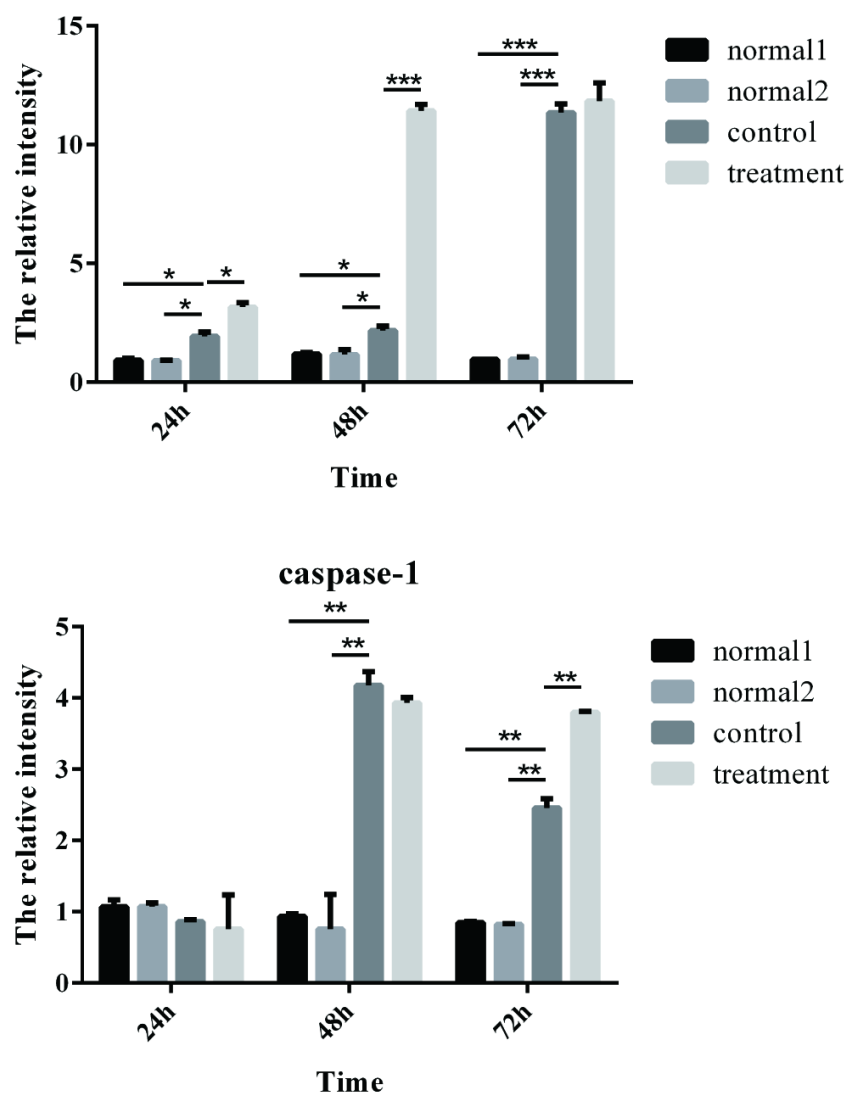

\section{Figure 3}

S. agalactiae increased the level of IL-1 $\beta$, NLRP3, caspase-1, and TNFRSF21 expression in mice. The mammary gland tissues of each group $(n \geq 3)$ were collected at $24 \mathrm{~h}, 48 \mathrm{~h}$, and $72 \mathrm{~h}$ after $\mathrm{S}$. agalactiae and PBS infection. a and e The level of IL-1 $\beta$, NLRP3, caspase-1, and TNFRSF21 protein expression in the mice was detected by Western blot. $b, c, d$, and $f$ The relative IL-1 $\beta$, NLRP3, caspase-1, and TNFRSF21 protein levels were quantified using Image $\mathrm{J}$ software and normalized to GAPDH expression in mice. Data are presented as the means \pm the standard deviation $(S D)(n \geq 3)\left({ }^{\star} P<0.05 ; * \star P<0.01 ; * \star * P<0.001\right)$. 

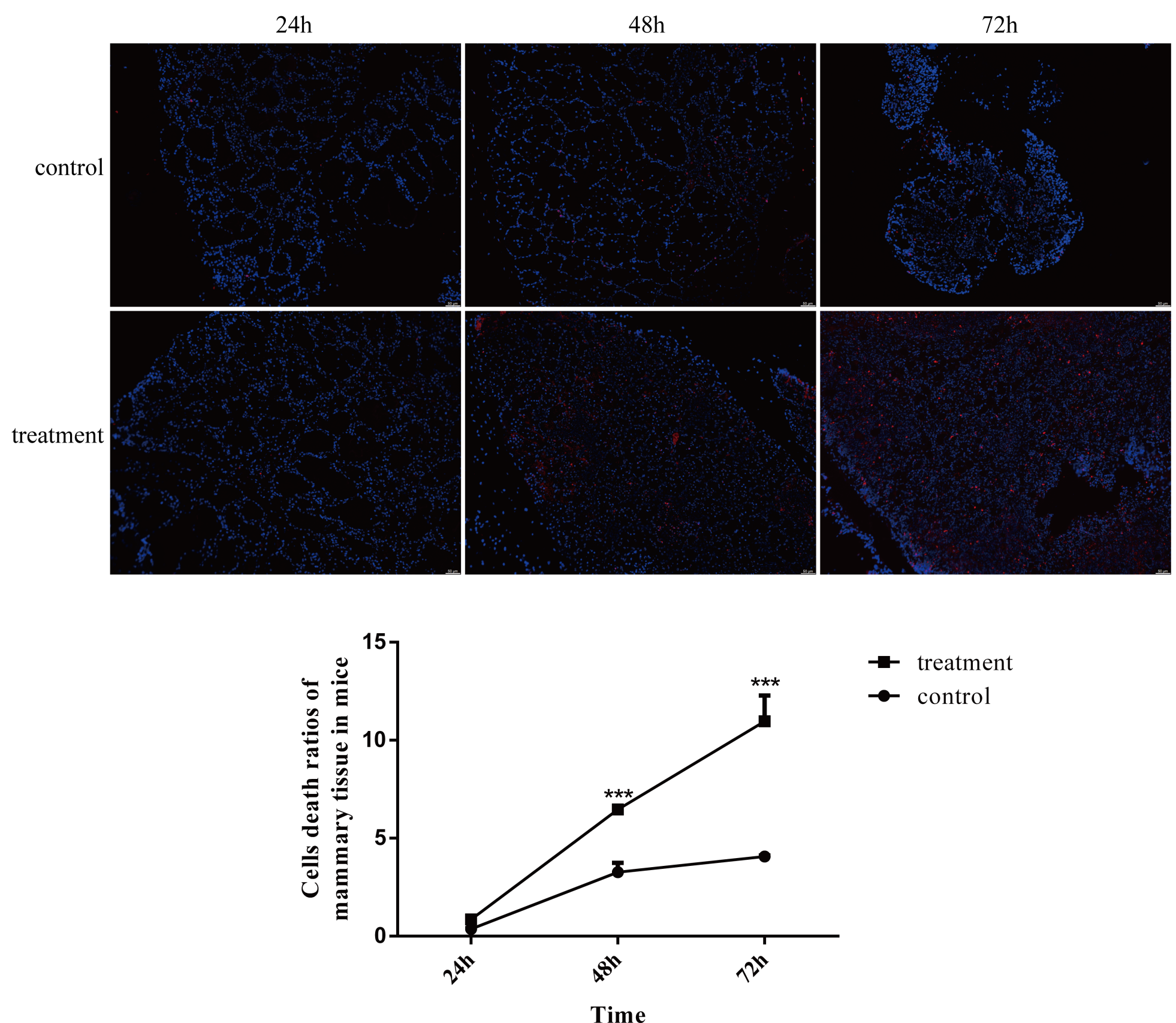

Figure 4

S. agalactiae induced pyroptosis in the mammary epithelial cells of the mice. a TUNEL staining of pyroptosis in the mouse mammary gland tissues in each group $(n \geq 3)$. b The percentage of pyroptosis in the mouse breast tissue. Data are presented as the means \pm the standard deviation $(S D)(n \geq 3)(* \star \star P<$ $0.001)$. 


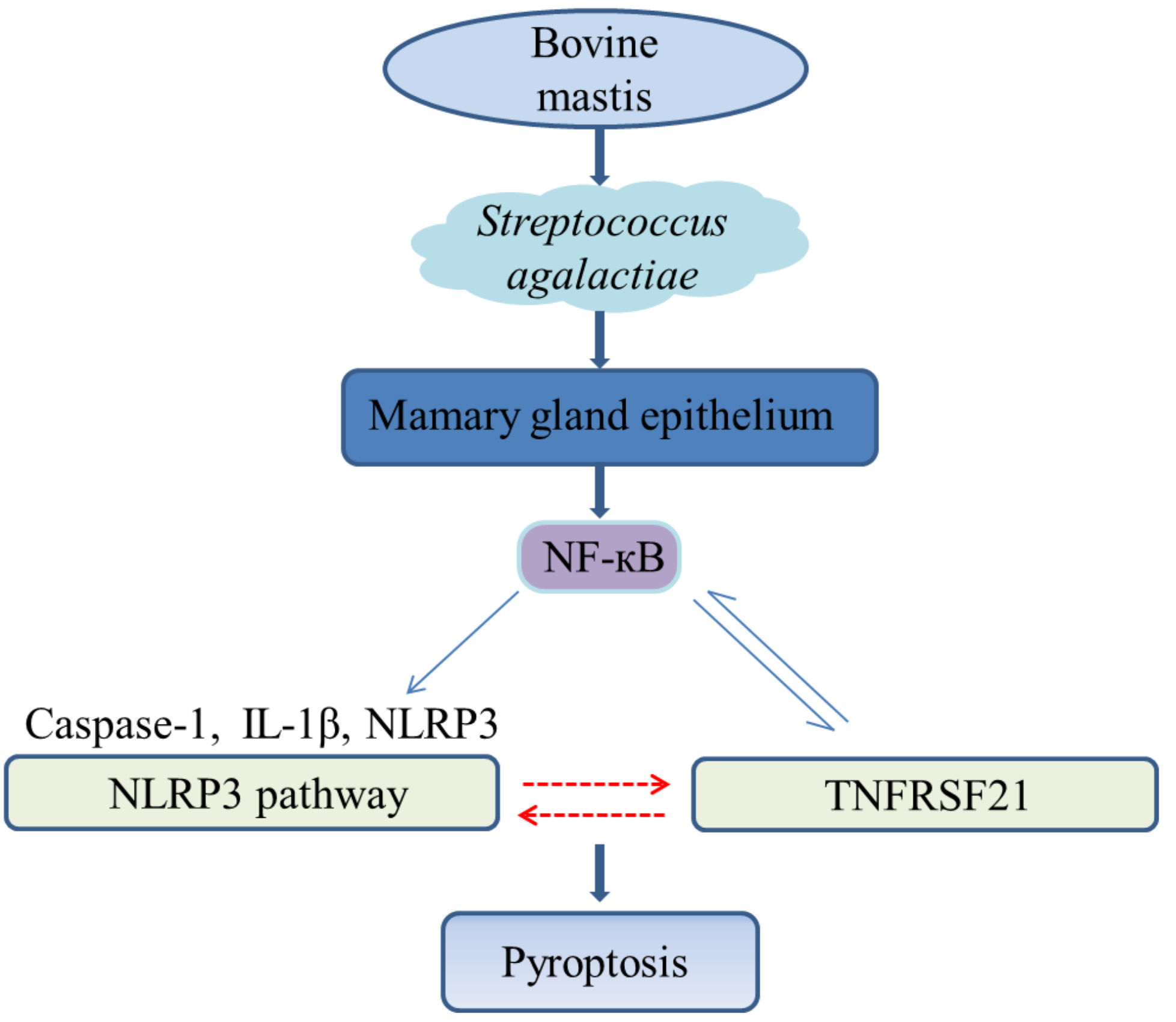

Figure 5

S. agalactiae-stimulated mammalian mammary gland epithelium-induced pyroptosis via activation of the NLRP3 pathway and excessive TNFRSF21 expression. S. agalactiae was isolated from bovine mastitis milk samples, which was used to infect the mice. The $\mathrm{S}$. agalactiae-infected mammary gland epithelium activated the NLRP3 inflammasome and induced excessive TNFRSF21 expression; however, there was no evidence to support the relationship between NLRP3 inflammasome activation and TNFRSF21. Finally, pyroptosis was observed in the mammary epithelial cells. 